\title{
Micropropagation for the Production of High Quality Phytochemicals
}

\author{
T. Grevenstuk and A. Romano \\ Institute for Biotechnology and Bioengineering \\ Centre of Genomics and Biotechnology (IBB/CGB) \\ Faculty of Sciences and Technology \\ University of Algarve \\ Campus de Gambelas \\ Ed. 8, 8005-139 Faro \\ Portugal
}

Keywords: in vitro biomass production, bioseparation, bioprospection, D. intermedia, plumbagin

\begin{abstract}
Plants area source of many valuable secondary metabolites that find a broad field of applications, ranging from the agrochemical to the pharmaceutical industries. Establishing a suitable source for extraction of phytochemicals is, however, not always straightforward. In many instances the production by chemical synthesis is not economically viable due to their complex structures and conservation issues may arise when they are harvested from natural sources. In vitro culture techniques offer an attractive alternative to these issues. Natural grown plants can be replaced by in vitro produced biomass with the advantage that several strategies can be implemented to increase production yields, such as genotype selection, altering growth conditions and use of elicitors, so that the higher investment costs are justified. Also, because plant tissue cultures can be generated on a continuous year-round basis without seasonal constraints, they can guarantee reliable and predictable production levels, which is of great importance for efficient process down-stream. Plant tissue culture techniques offer the possibility of establishing cultures from leaves, stems, roots and meristems, meaning that metabolites produced in specific plant organs can also be prospected. The successful production of a large number of phytochemicals from micropropagated biomass has been reported, and it seems that only in a few cases cultures fail to accumulate compounds of interest. The advantages and the range of possibilities offered by plant tissue culture techniques suggest that these might become a valuable and indispensable tool for the production of phytochemicals. In this work, the example of the prospection of plumbagin from micropropagated $D$. intermedia plants is described. Plumbagin is a naphthoquinone with potential pharmaceutical applications and results obtained by several hyphenated analytical techniques confirm that an end product with high purity and recoveries can be obtained from in vitro cultured plants.
\end{abstract}

\section{INTRODUCTION}

The Importance of the Plant Kingdom

Many specialty materials that find application in the pharmaceutical, food, cosmetic and agrochemical industries are obtained from plants. It is estimated that plantderived natural products represent more than $25 \%$ of all drugs in clinical use in the world (Rates, 2000; Gurib-Fakim, 2006). Examples of important drugs obtained from plants are digoxin from Digitalis spp., quinine and quinidine from Cinchona spp., vincristrine and vinblastine from Catharanthus roseus, atropine from Atropa belladonna and morphine and codeine from Papaver somniferum (Rates, 2000). However, the potential of higher plants as a source for new drugs is still largely unexplored. Among the estimated 250000500000 plant species, only a small percentage has been investigated chemically and the fraction submitted to biological or pharmacological screening is even smaller (Hamburger 
and Hostettmann, 1991). In this context the progressive loss of biodiversity is alarming, as it is predicted that $25 \%$ of today's flowering plant species will be lost by 2025 (Houghton, 2001). Regarding the field of herbal medicine, plant derived extracts, teas or capsules, designated more generically as phytomedicines or botanical drugs, are widely used in many European countries and USA, where they are produced according to high standards and submitted to thorough clinical trials (Lubbe and Verpoorte, 2011). Examples of popular phytopharmaceuticals are Gingko biloba, Hypericum perforatum (St. John's Wort) and Valeriana officinalis (Valerian) extracts (Houghton, 2001). It is difficult to assess the global turnover of plant derived fine chemicals on a yearly basis, but the figures concerning the exportation and importation of medicinal and aromatic plants alone amounted to considerable values (Table 1) in the period of 2004-2008. Also, many plant species are in demand for dye extraction due to an increasing popularity of natural dyes. Tagetes patula, Juglans regia, Lawsonia inermis are examples of plants from which dyes are obtained. Other species are sources of valuable materials for the manufacture of cosmetic products, such as oils, fats and waxes, essential oils and oleoresins and plant extracts. In the agrochemical sector, many insect, microbial pathogens and weed control agents are obtained from plants. Some examples are nicotine isolated from Nicotiana spp., karanjin from Derris indica and eugenol extracted from Eugenia caryophyllus (Lubbe and Verpoorte, 2011).

\section{Sources of Plant Material: from Wild Collection to In Vitro Production}

In the case of pharmaceutical and other large industries, cultivation is usually done under contract to ensure a reliable supply of material but also to allow higher standardization of raw materials. However, in most cases raw materials are harvested from nature due to lower investment requirements. When raw material is obtained from widely occurring, fast growing species whose foliage alone is harvested, over-harvesting does not have to be an issue. However, when dealing with slow-growing species with limited distribution and when obtaining the end product involves harvesting the roots or bark, over-harvesting and consequently conservation problems may arise (Lubbe and Verpoorte, 2011). As an alternative, the pharmaceutical industry has recurred to chemical synthesis to produce many plant-derived pharmaceuticals, which has largely been successful. However, in some cases this has proved to be uneconomic in comparison to isolation from plant material. Camptothecin (Camptotheca acuminata), podophyllotoxin (Podophyllum hexandrum), vinblastine and vincristine are examples of anticancer compounds that are extracted from their natural sources and not synthesized chemically on a commercial scale due to their complex structure with several chiral centres (Wink et al., 2005). Also, the fact that many plant species are not easy to cultivate and have specific climatic requirements, has as repercussion inconsistent production and low yields, making the approach of cultivation unfeasible in the long term (Georgiev et al., 2009).

In response to these issues, in vitro culture technology has become an attractive and cost-effective alternative for the production of high value plant-derived metabolites. Plant cell and tissue cultures can be established routinely under sterile conditions from explants, such as plant leaves, stems, roots and meristems for both multiplication and extraction of chemicals, as they produce and accumulate many of the same valuable compounds as the parent plant in nature. Secondary metabolite production in plant cell and tissue culture has the advantage of being reliable, predictable, generated on a continuous year-round basis without seasonal constraints, and in some cases yields may exceed the ones found in nature (Debnath et al., 2006; Karuppusamy et al., 2009). As an alternative, this technology can be used to produce plantlets at large scale for cultivation. For instance, the sexual reproduction of Gingko biloba is compromised by the recalcitrant nature of its seeds, which has encouraged many research projects towards the production of plantlets by means of in vitro culture techniques (Tommasi and Scaramuzzi, 2004; Uchendu et al., 2011). However, considering the high investment of in vitro culture in comparison to traditional cultivation, it is expected that these techniques will only be 
employed for the production of phytochemicals with added value.

In a limited number of instances production of high-value phytochemicals in non-organized callus and suspension cultures using bioreactors was successfully implemented. Paclitaxel is an alkaloid that was first isolated from the bark of Taxus brevifolia, during a screening program of the United States National Cancer Institute, and is currently produced by plant cell fermentation by Phyton Biotech (Germany). Sold under the trademark Taxol $\AA$, paclitaxel is used in cancer chemotherapy to treat lung, ovarian and breast cancer and is a good example of commercially viable production of secondary metabolites using biotechnological approaches (Wink et al., 2005).

However, undifferentiated callus and suspension cultures very often fail to accumulate the compounds of interest. This situation occurs when the metabolite is only produced in specialized plant tissues or glands in the parent plant and production requires more differentiated microplant or organ cultures. A prime example is the production of ginseng saponins (Panax ginseng), for which root culture is required as these saponins are produced specifically in the roots (Karuppusamy, 2009). On the other hand, organized tissue cultures such as shoot and root cultures, as well as hairy roots, hardly ever fail to synthesize secondary metabolites. Karuppusamy (2009) presents an extensive review on the metabolites produced by in vitro tissue, organ and cell cultures, showing the potential of these techniques to produce the most diverse phytochemicals.

\section{CASE STUDY: PLUMBAGIN RECOVERY FROM DROSERA INTERMEDIA}

The following section describes the research effort developed at the Plant Biotechnology Laboratory (University of Algarve) to reach a chemically well-defined phytochemical from in vitro reared plants, ranging from the development of a micropropagation protocol, to the process development for the extraction and purification of plumbagin from $D$. intermedia.

\section{D. intermedia}

Drosera intermedia (H.) Hayne, or spoonleaf sundew, is a perennial herb that forms a semi-erect stemless rosette of spatulate leaves (Fig. 1A) and is typically found in nutrient-poor peat lands including rain-fed bogs and poor fens (Juniper et al., 1989). The genus Drosera (Droseraceae) comprises nearly 150 species with a worldwide distribution. However, the vast majority of species are found in the Southern Hemisphere, especially in south-western Australia (Rivadavia et al., 2003). In Europe only three species exist, Drosera anglica, D. intermedia and D. rotundifolia (Crowder et al., 1990). Plants of the Drosera genus bear leaves with pin-shaped tentacles covered at the tip with glistering drops of mucilage that resemble drops of morning dew and to which they owe their scientific name (from Greek droseros, dewy) and their common name of sundew (Crowder et al., 1990). Drosera species have long held the interest of botanists and horticulturists because of their unique biology and carnivorous habit. However, apart from their ornamental value, Drosera plants have medicinal significance and due to uncontrolled collection, natural populations are becoming increasingly scarce, especially in Europe where the Drosera species are included in the European Red List of Threatened Plants (Kawiak et al., 2003). D. intermedia produces self compatible flowers and is also able to reproduce vegetatively by production of side rosettes during the growing season, regeneration of plantlets on senescing tissue and formation of axillary buds in autumn (de Ridder and Dhondt, 1992). D. intermedia is known to produce plumbagin (Budzianowski, 1996) but the biochemical description of this species in literature is poor. $D$. intermedia has been used as an infusion or a tincture for asthma, pulmonary catarrh and whooping cough (Crowder et al., 1990).

\section{The Naphthoquinone Plumbagin}

Plumbagin (5-hydroxy-2-methyl-1,4-naphthalenedione; Fig. 2) is the most efficient secondary metabolite isolated so far from carnivorous plants (Eilenberg et al., 2005). This naphthoquinone has received an enormous amount of attention in 
pharmacological research due to its antimalarial (Likhitwitayawuid et al., 1998), antimicrobial (Didry et al., 1994, 1998), antifungal (Ribeiro de Paiva et al., 2003), anticancer (Parimala and Sachdanandam, 1993; Sugie et al., 1998), antimutagenic (Durga et al., 1992), cardiotonic (Itoigawa et al., 1991), hypolipidemic and antiatherosclerotic effects (Sharma et al., 1991). Despite the broad range of biological activities of plumbagin, its high cytotoxicity and relative low therapeutic selectivity are the major disadvantages that limit its medical application (Kayser et al., 2003). To circumvent this problem several synthetic plumbagin derivatives have been examined to identify products exerting plumbagin-like activity and lower toxicity (Ogihara et al., 1997; Hazra et al., 2002; Tandon et al., 2005). Nevertheless, it has been shown recently that at subtoxic concentrations, plumbagin is proving to be an effective agent against several pharmacological targets and has regained the interest of researchers in the field of drug discovery in the past years (Shieh et al., 2010; Luo et al., 2010) (Fig. 3). Furthermore, the inhibition of insect ecdysis and chitin synthetase by plumbagin suggests that plumbagin and its closely related derivatives may serve as environmentally friendly insect control agents and be of value to the agrochemical industry (Kubo et al., 1983).

\section{Occurrence and Biological Significance of Plumbagin}

Naphthoquinones are one of the groups of secondary metabolites widespread in nature. Plumbagin is produced by a disperse and heterogeneous group of plant families and can be found in members of the Droseraceae (Marckzack et al., 2005), Plumbaginaceae (Ribeiro de Paiva et al., 2003), Nepenthaceae (Rischer et al., 2002), Ebenaceae (Dzoyem et al., 2007) and also in Drosophyllum lusitanicum (Grevenstuk et al., 2008) and Triphyophyllum peltatum (Bringmann et al., 2000), which belong to the monotypic families Drosophyllaceae and Dioncophyllaceae, respectively. The production of naphthoquinones is common in carnivorous plants but not exclusive to this group of plants. The ability of naphthoquinone synthesis is not limited to higher plants. Naphthoquinones have also been found in fungal organisms in genera such as Aspergillus, Cladosporium, Fusarium, Microsporium, Mollisia, Penicillium, Trichophyton and Verticillium and Actinomycetes of the genus Streptomyces (Medentsev and Akimenko, 1998). Plumbagin, however, has not been found in these organisms to date.

Besides the effects of plumbagin on pharmacological targets, there are many reports on its allelochemical effects on organisms of interest in the ecological context. Plumbagin acts as an antifeedant agent on herbivorous insects, including adapted Lepidoptera (Villavicencio and Perez-Escandon, 1994), is responsible for fungicidal activity against phytopathogenic species, acts as a potent phytoalexin against parasitic plants (Bringmann et al., 1999) and inhibits the germination of seeds from other species (Gonçalves et al., 2009). These findings support the importance of the production of plumbagin and related naphthoquinones, improving the plants' fitness and conferring an adaptive advantage over other plants (Rischer et al., 2002).

\section{The Exploitation of Plumbagin}

At present, the most exploited source of plumbagin are the roots of Plumbago spp. However, these plants grow quite slowly and the roots suitable for extraction take years to grow (Komaraiah et al., 2003). Moreover, conventional propagation of the plant is rather difficult and insufficient to meet the growing demand, owing to the poor germination of seeds and death of young seedlings under natural conditions (Verma et al., 2002). Attempts to produce plumbagin synthetically proved to be commercially ineffective (Ichihara et al., 1980; Wurm and Gurka, 1986), and therefore alternatives for the production of plumbagin based on in vitro techniques should be sought.

Nahálka et al. (1996) showed that cell suspension cultures of D. lusitanicum are capable of producing large quantities of plumbagin in a short amount of time. However, vitality of the suspension was relatively low because of cell plasmolysis and compromised the process viability (Nahálka et al., 1998). Plumbagin is produced mainly 
in the roots of Plumbago spp., therefore, researchers used another approach using hairy root cultures in the expectation that it might lead to high levels of plumbagin production (Verma et al., 2002; Gangopadhyay et al., 2008). Hairy roots are obtained by transforming root cultures with Agrobacterium rhizogenes, resulting in a phenotype that is characterized by fast hormone-independent growth, lack of geotropism and genetic stability (Veena and Taylor, 2007). Despite achieving promising biomass production rates, the plumbagin content was too low for commercial exploitation.

Komaraiah et al. (2003) used an improved method to produce plumbagin using Plumbago indica (same species as $P$. rosea) cell cultures. The cells were immobilized in calcium alginate while elicitated using chitosan and plumbagin was recovered simultaneously using in situ adsorption. Elicitors are signal compounds of plant defence responses, which can therefore enhance the production of secondary metabolites. The most frequently used elicitors are fungal carbohydrates, yeast extract and chitosan (Karuppusamy, 2009). The immobilized cell system enhanced plumbagin production, possibly due to increased degree of differentiation or cell-to-cell contact (Komaraiah et al., 2001). In situ direct extraction also increased plumbagin recoveries by reducing the toxic effects of plumbagin on the cells and feedback inhibition of secondary metabolite synthesis. However, Plumbago plants might not be the most adequate source of plant material for the bioprospection of plumbagin because its content in field specimens is lower when compared to other plumbagin bearing plants, such as $D$. lusitanicum for instance (Grevenstuk et al., 2008). Drosera spp. were never seriously considered for plumbagin prospection due to their low biosynthesis of this naphthoquinone (Crouch et al., 1990), nevertheless, this issue is controversial since higher recoveries have been obtained from Drosera plants in comparison to Plumbago plants (Marczak et al., 2005; Krolicka et al., 2008; Putalun et al., 2010).

\section{Bioprospection of Plumbagin from Micropropagated $D$. intermedia}

1. Micropropagation of $D$. intermedia and Evaluation of Biomass Production. A micropropagation protocol to produce $D$. intermedia biomass at large scale was developed in order to optimize the plumbagin extraction procedure (Grevenstuk et al., 2010). In brief, initial explants were obtained from seedlings (Fig. 1B) germinated in 1/4 Murashige and Skoog (1962; MS) medium under a 16-h photoperiod at a temperature of $25 \pm 2^{\circ} \mathrm{C}$. Seedlings were sub-cultured onto media with three concentrations of MS macro-nutrients (total MS, $1 / 2 \mathrm{MS}$ and $1 / 4 \mathrm{MS}$ ) supplemented with $0.1 \mathrm{mg} \mathrm{dm}^{-3}$ of kinetin or without plant growth regulators and after 8 weeks of culture proliferation and rooting parameters were evaluated. The results showed that proliferation was not affected by addition of kinetin but was significantly influenced by MS macronutrient concentration. The highest proliferation was obtained in $1 / 4 \mathrm{MS}$ medium, which is in agreement with results obtained for other carnivorous plants and seems to be a characteristic to this group (Jang et al., 2003; Kim and Jang, 2004; Gonçalves et al., 2005). The rooting capacity of shoots was barely influenced by the variables introduced in the experiment as rooting percentages of $100 \%$ were obtained in nearly all cases. Either way, this parameter is of less importance since the main purpose of the protocol is to obtain high biomass production levels. Therefore, the proliferation rate is preponderant in this case and the best medium to produce $D$. intermedia biomass was found to be $1 / 4 \mathrm{MS}$ medium without plant growth regulators (Fig. 1C).

To determine the optimum harvesting period, the biomass increment of $D$. intermedia cultures was monitored over time. The culture growth index was calculated as the fresh weight gain divided by the culture's initial fresh weight and was registered at 2 week intervals during 16 weeks of culture. Separate cultures with the same culture time as the ones used for plumbagin extraction were dried until constant weight to determine its water content for dry weight yield determination. The growth curve obtained for $D$. intermedia cultures (Fig. 4) shows that the biomass increases slowly during the first 6 weeks of culture, period after which the cultures grow more vigorously, reaching a $9.70 \pm$ 0.94 fold increase in biomass after 10 weeks of culture. The efficiency of in vitro culture 
methods is usually evaluated in terms of the capacity of an explant to generate new shoots, instead of biomass production. It is therefore difficult to assess the efficiency of $D$. intermedia biomass production in the tested growth conditions, as these results are available only for few Drosera species. However, $D$. intermedia does seem to grow more vigorously than Drosera capensis. After 4 weeks of growth in $1 / 2 \mathrm{MS}$ medium, biomass gain of $D$. capensis control cultures amounted to $1.193 \pm 0.035 \mathrm{~g} \mathrm{FW} / \mathrm{g}$ initial FW (Krolicka et al., 2008), while for D. intermedia a gain of $2.79 \pm 0.10 \mathrm{~g} \mathrm{FW} / \mathrm{g}$ initial FW (result not shown; $1.74 \pm 0.10$ fold biomass increment) was scored. The same author also reported a two-fold increase in biomass production after addition of jasmonic acid, meaning that the biomass production of $D$. intermedia could be further enhanced.

The biosynthesis of plumbagin was not monitored over time, which could lead to misassumptions concerning the optimum harvesting time, as the production of plumbagin can vary depending on the growth phase. However, Verma et al. (2002) showed that the highest plumbagin concentration found in the roots of $P$. zeylanica hairy cultures coincided with the period with the highest biomass production. The authors reported a 21 fold increase in biomass yield after 6 weeks of culture. The higher biomass production yields reported for $P$. zeylanica cultures are expectable since hairy roots have higher growth rates and no growth regulators were used when producing $D$. intermedia cultures.

2. Optimization of Plumbagin Extraction. To evaluate the plumbagin bioproduction levels of $D$. intermedia, several extraction techniques were employed, including maceration under mechanical stirring, Soxhlet extraction (SE), Ultrasound Assisted Extraction (UAE) and Supercritical Fluid Extraction (SFE). Solvent extractions were performed with $n$-hexane using the same volume of solvent to compare extraction efficiency and supercritical $\mathrm{CO}_{2}$ without modifiers was the solvent of choice for SFE. The results were recently published (Grevenstuk et al., 2012) and indicated that the amount of plumbagin that can be recovered from $D$. intermedia is considerable. Table 2 summarizes the obtained recoveries and shows that as much as $3.12 \pm 0.05 \mathrm{mg} / \mathrm{g} \mathrm{FW}$ can be obtained when using UAE.

Although all extractions were performed with fresh plant material, the content in plumbagin is presented on fresh weight and dry weight basis in order to allow comparison with previous publications. Despite the fact that most extraction methods use dry plant material, for the purpose of this work the extractions were performed with fresh plant material because the extraction of plumbagin is greatly hampered when using dry material (Verma et al., 2002; Marczak et al., 2005; Grevenstuk et al., 2008). Marczak (2005) hypothesized that plumbagin may be more strongly bound to the dry plant matrix instead of being decomposed during the drying process. The author showed that it was possible to extract plumbagin from dried material with methanol, even after it had been extracted with chloroform, possibly due to easier disruption of intermolecular interactions with the more polar solvent. When performing extractions with fresh samples, higher yields were obtained with chloroform than with the other solvents.

3. D. intermedia as a Source of Plumbagin. As mentioned earlier, plumbagin is currently exploited from plants of the Plumbago genus. However, when comparing the recoveries presented in Table 2 with the contents in plumbagin of Plumbago spp. reported in literature, one can conclude that $D$. intermedia could be an alternative source of plumbagin. The content in plumbagin obtained by maceration with ethyl acetate of dried P. zeylanica roots varies between 0.629 and $4.975 \mathrm{mg} / \mathrm{g}$ according to Wang et al. (2005). For the same species, Hsieh (2005) reported a recovery of $13.40 \pm 1.30 \mathrm{mg} / \mathrm{g} \mathrm{DW}$ after extracting the plant material with boiling ethanol. Another author reported recoveries of $1.50,1.91$ and $1.40 \mathrm{mg} / \mathrm{g}$ DW obtained from roots of Plumbago auriculata, P. indica and $P$. zeylanica, respectively, obtained by SE with acetone (Mallavadhani et al., 2002). Despite the fact that in these reports the plant material was dried prior to extraction and the extraction procedures are diversified, the recoveries are in some cases considerably lower than those obtained from $D$. intermedia. The lowest plumbagin recovery was 17.51 $\pm 1.40 \mathrm{mg} / \mathrm{g} \mathrm{DW}$, obtained by maceration. Roots of $P$. scandens were extracted by SFE with plumbagin contents ranging from 0.056 to $1.93 \mathrm{mg} / \mathrm{g} \mathrm{FW}$ depending on the time 
period between collection and extraction (Rodrigues et al., 2006). Using the same extraction method and experimental conditions, $2.54 \pm 0.14 \mathrm{mg} / \mathrm{g} \mathrm{FW}$ were obtained from $D$. intermedia. It is worth underlining that the results mentioned above were obtained from field specimens of Plumbago spp. and might therefore be overestimated. The differences of the recoveries reported in literature for plants of the Plumbago genus, even for those of the same species, are in part due to the different extraction procedures but also due to geographical and seasonal factors which affect the content in secondary metabolites, underlining one of the advantages of using in vitro cultures for bioprospection, as it is a more reliable and qualitatively consistent source of plant material. In fact, by comparing the presented experimental deviations obtained in a similar study conducted with field specimens of D. lusitanicum (Grevenstuk et al., 2008) one can conclude that issues of material homogeneity are greatly reduced when using micropropagated plant material.

Several biotechnological approaches have been used to improve the production yield of plumbagin of Plumbago spp., while simultaneously preventing harvesting of the whole plant. Hairy root cultures have been established from P. zeylanica (Verma et al., 2002) and P. indica (Gangopadhyay et al., 2008) with the intent of increasing the growth rate and plumbagin production. The hairy root cultures resulted in increased plumbagin yields in comparison to untransformed roots $(8.40 \mathrm{mg} / \mathrm{g}$ DW from $P$. zeylanica; 6.18 $\mathrm{mg} / \mathrm{g}$ DW from $P$. indica), however, the obtained plumbagin contents are inferior to those obtained from $D$. intermedia cultures (Table 2). Another approach used to improve the production of plumbagin was to immobilize cell cultures of $P$. indica in calcium alginate while being elicited with chitosan and collecting plumbagin by in situ adsorption, thereby reducing the feedback inhibition of secondary metabolite production (Komaraiah et al., 2003). This way, recoveries of $92.13 \mathrm{mg} / \mathrm{g}$ of dry cell weight (DCW) were obtained, which is nearly four times more than the highest recovery obtained from $D$. intermedia $(24.78 \pm 0.55 \mathrm{mg} / \mathrm{g} \mathrm{DW})$. In the cited study, the step of plumbagin elicitation with chitosan alone was responsible for a production increment of over six times, meaning that the production of plumbagin by $D$. intermedia could be enhanced considerably as well using adequate elicitors. In a different study the production of plumbagin by Drosera burmanii suffered a 3.5-fold increase over control due to elicitation by yeast extract application (Putalun et al., 2010).

$D$. intermedia produces significant amounts of plumbagin, even when compared to plants of the same genus. D. intermedia seems to produce higher levels of the naphthoquinone then the eight in vitro cultured Drosera species evaluated by Marczak (2005). In this study, the highest plumbagin recovery was obtained from D. binata (12.4 $\mathrm{mg} / \mathrm{g} \mathrm{DW}$ ) using UAE with chloroform as extracting solvent. Using the same extraction technique, higher recoveries were obtained from $D$. intermedia $(24.78 \pm 0.55 \mathrm{mg} / \mathrm{g} \mathrm{DW})$. It has to be stated that chloroform can give higher recoveries, although this increment usually comes at cost of the extract purity, because a greater amount of undesired compounds are co-extracted (Grevenstuk et al., 2008).

The plumbagin production levels of $D$. intermedia seem to be similar to those reported for D. lusitanicum (Grevenstuk et al., 2008). Field specimens were extracted with $n$-hexane using SE and UAE affording recoveries of $2.42 \pm 0.39 \mathrm{mg} / \mathrm{g} \mathrm{FW}$ and 1.52 $\pm 0.39 \mathrm{mg} / \mathrm{g} \mathrm{FW}$, respectively. The recovery of plumbagin using SE is comparable to the one obtained from $D$. intermedia $(2.67 \pm 0.04 \mathrm{mg} / \mathrm{g} \mathrm{FW})$. The plumbagin content of the extract obtained by UAE is lower compared to the one obtained from $D$. intermedia $(3.12$ $\pm 0.05 \mathrm{mg} / \mathrm{g} \mathrm{FW}$ ), but this can be explained by the fact that an experimental setup with a horn transducer was used to deliver ultrasounds to the D. lusitanicum matrix, instead of an ultrasound bath. In this setup, the ultrasounds are only delivered efficiently to the plant matrix close the horn transducer, leading to lower recoveries. In a different work, cell suspension cultures were established from D. lusitanicum, yielding high amounts of plumbagin (35 mg/g Fresh Cell Weight) (Nahálka et al., 1996). The cell suspension culture was able to produce over 10 times more than the mother plant, but the cultures underwent a strong plasmolysis short after being transferred to the liquid medium, 
making this approach unviable. D. lusitanicum is a species endemic to the Iberian Peninsula and northern Morocco and is in risk of eminent extinction; therefore harvesting this plant from the wild for plumbagin extraction is unfeasible and irresponsible from an ecological perspective. An in vitro culture protocol has been developed for D. lusitanicum (Gonçalves et al., 2005), but D. intermedia seems to grow easier and more vigorously in these conditions.

4. Plumbagin Identification and Purity Determination. In order to confirm that plumbagin was in fact being extracted, a sample was cleaned using a solid phase extraction (SPE) column and analysed by Mass Spectrometry and Nuclear Magnetic Resonance experiments (NMR). The negative-ion ESI-MS spectrum showed only one major peak with a pseudo-molecular ion peak $[\mathrm{M}-\mathrm{H}]^{-}$at $\mathrm{m} / \mathrm{z}$ (mass to charge ratio) 187 , suggesting a molecular formula of $\mathrm{C}_{11} \mathrm{H}_{8} \mathrm{O}_{3}$. Because the extract was very concentrated, a good quality Heteronuclear Multiple Bond Correlation (HMBC) spectrum was obtained and by comparison with literature data the main compound of the extract could readily be identified as plumbagin (Sankaram et al., 1986). The obtained ${ }^{13} \mathrm{C}$ and ${ }^{1} \mathrm{H}$ NMR spectra pointed out a lack of signals belonging to contaminating compounds, indicating that the analysed sample is practically pure. Subsequently, the samples obtained by different extraction methods were cleaned with the same SPE procedure as well and analysed by HPLC for purity determination. The obtained results were remarkable as in some cases purities over $99.5 \%$ were achieved (Table 3 ). The products obtained from the SE and UAE extracts were the most pure $(P<0.05)$, with purities of $99.91 \pm 0.09 \%$ and $99.51 \pm$ $0.49 \%$, respectively. Another positive result was that the product recovery of the process was relatively high $(86.31 \%$ for extract obtained by SFE) meaning that $2.74 \mathrm{mg}$ of plumbagin ( $>99 \%$ purity) can be obtained from $1 \mathrm{~g}$ of micropropagated plant material (Grevenstuk et al., 2012).

\section{CONCLUSIONS}

The presented results show that high purity plumbagin can be produced sustainably from $D$. intermedia cultures with high yields. These results are an example of an application of in vitro culture techniques for the production of fine chemicals. D. intermedia cultures grow fast and have high levels of plumbagin bioproduction and could therefore represent an alternative for the bioprospection of plumbagin. Taking into consideration that no growth regulators or elicitors were used it is possible that the production of plumbagin can be further enhanced. Furthermore, extracting plant metabolites from in vitro cultures is desirable when high yields can be obtained, because plant materials are not completely homogeneous and are affected by seasonal and geographical factors. Micropropagated plant material is more reliable and homogeneous and this might enable the scale-up of an efficient process amenable to industry.

\section{ACKNOWLEDGEMENTS}

T. Grevenstuk acknowledges a grant from the Portuguese Science and Technology Foundation (FCT, Grant SFRH/BPD/73293/2010).

\section{Literature Cited}

Bringmann, G., Rischer, H., Wohlfarth, M., Schlauer, J. and Assi, L.A. 2000. Droserone from cell cultures of Triphyophyllum peltatum (Dioncophyllaceae) and its biosynthetic origin. Phytochemistry 53:339-343.

Bringmann, G., Schlauer, J., Rückert, M., Wiesen, B., Ehrenfeld, K., Proksch, P. and Czygan, F.C. 1999. Host-derived acetogenins involved in the incompatible parasitic relationship between Cuscuta reflexa (Convolvulaceae) and Ancistrocladus heyneanus (Ancistrocladaceae). Plant Biol. 1:581-584.

Budzianowski, J. 1996. Naphthohydroquinone glucosides of Drosera rotundifolia and $D$. intermedia from in vitro cultures. Phytochemistry 42:1145-1147.

Crouch, I.J., Finnie, J.F. and vanStaden, J. 1990. Studies on the isolation of plumbagin from in vitro and in vivo grown Drosera species. Plant Cell Tiss. Org. Cult. 21:79-82. 
Crowder, A.A., Pearson, M.C., Grubb, P.J. and Langlois, P.H. 1990. Drosera L. J. Ecol. 78:233-267.

de Ridder, F. and Dhondt, A.A. 1992. The reproductive behaviour of a donal herbaceous plant, the longleaved sundew Drosera intermedia, in different heathland habitats. Drosera intermedia, in different heathland habitats. Ecography 15:144-153.

Debnath, M., Malik, C.P. and Bisen, P.S. 2006. Micropropagation: A tool for the production of high quality plant-based medicines. Current Pharmaceutical Biotechnology 7:33-49.

Didry, N., Dubreuil, L. and Pinkas, M. 1994. Activity of anthraquinonic and naphthoquinonic compounds on oral bacteria. Pharmazie 49:681-683.

Didry, N., Dubreuil, L., Trotin, F. and Pinkas, M. 1998. Antimicrobial activity of aerial parts of Drosera peltata Smith on oral bacteria. J. Ethnopharmacol. 60:91-96.

Durga, R., Sridhar, P. and Polasa, H. 1992. Antimutagenic activity of plumbagin in Ames Salmonella typhimurium test. Ind. J. Med. Res. 96:143-145.

Dzoyem, J.P., Tangmouo, J.G., Lontsi, D., Etoa, F.X. and Lohoue, P.J. 2007. In vitro antifungal activity of extract and plumbagin from the stem bark of Diospyros crassiflora Hiern (Ebenaceae). Phytother. Res. 21:671-674.

Eilenberg, H., Pnini-Cohen, S., Rahamim, Y., Sionov, E., Segal, E., Carmeli, S. and Zilberstein, A. 2010. Induced production of antifungal naphthoquinones in the pitchers of the carnivorous plant Nepenthes khasiana. J. Exp. Bot. 61:911-922.

Gangopadhyay, M., Sircar, D., Mitra, A. and Bhattacharya, S. 2008. Hairy root culture of Plumbago indica as a potential source for plumbagin. Biol. Plant. 52:533-537.

Georgiev, M.I., Weber, J. and Maciuk, A. 2009. Bioprocessing of plant cell cultures for mass production of targeted compounds. Appl. Microbiol. Biot. 83:809-823.

Gonçalves, S., Ferraz, M. and Romano, A. 2009. Phytotoxic properties of Drosophyllum lusitanicum leaf extracts and its main compound plumbagin. Sci. Hortic. 122:96-101.

Gonçalves, S. and Romano, A. 2005. Micropropagation of Drosophyllum lusitanicum (Dewy pine), an endangered West Mediterranean endemic insectivorous plant. Biodivers. Conserv. 14:1071-1081.

Grevenstuk, T., Coelho, N., Gonçalves, S. and Romano, A. 2010. In vitro propagation of Drosera intermedia in a single step. Biol. Plant. 54:391-394.

Grevenstuk, T., Gonçalves, S., Nogueira, J.M.F., Bernardo-Gil, M.G. and Romano, A. 2012. Recovery of high purity plumbagin from Drosera intermedia. Ind. Crop. Prod. 35:257-260.

Grevenstuk, T., Goncalves, S., Nogueira, J.M.F. and Romano, A. 2008. Plumbagin recovery from field specimens of Drosophyllum lusitanicum (L.) Link. Phytochem. Anal. 19:229-35.

Gurib-Fakim, A. 2006. Medicinal plants: traditions of yesterday and drugs of tomorrow. Mol. Aspects Med. 27:1-93.

Hamburger, M. and Hostettmann, K. 1991. Bioactivity in plants: the link between phytochemistry and medicine. Phytochemistry 30:3864-3874.

Hazra, B., Sarkar, R., Bhattacharyya, S., Ghosh, P.K., Chel, G. and Dinda, B. 2002. Synthesis of plumbagin derivatives and their inhibitory activities against Ehrlich ascites carcinoma in vivo and Leishmania donovani Promastigotes in vitro. Phytother. Res. 16:133-137.

Houghton, P.J. 2001. Old yet new-pharmaceuticals from plants. J. Chem. Educ. 78:175184.

Hsieh, Y.J., Lin, L.C. and Tsai, T.H. 2005. Determination and identification of plumbagin from the roots of Plumbago zeylanica L. by liquid chromatography with tandem mass spectrometry. J Chromatogr. A 1083:141-145.

Ichihara, A., Ubukata, M. and Sakamura, S. 1980. Synthesis of plumbagin by the retroDiels-Alder reaction. Agr. Biol. Chem. 44:211-213.

Itoigawa, M., Takeya, K. and Furukawa, H. 1991. Cardiotonic action of plumbagin on guinea-pig papillary muscle. Planta Med. 57:317-319.

Jang, G.-W., Kim, K.-S. and Park, R.-D. 2003. Micropropagation of Venus fly trap by 
shoot culture. Plant Cell Tissue Organ Cult. 72:95-98.

Joshi, N.K. and Sehnal, F. 1989. Inhibition of ecdysteroid production by plumbagin in Dysdercus cingulatus. J. Insect Physiol. 35:737-741.

Juniper, B.E., Robins, R.J. and Joel, D.M. 1989. The Carnivorous Plants. Academic Press, London.

Karuppusamy, S. 2009. A review on trends in production of secondary metabolites from higher plants by in vitro tissue, organ and cell cultures. Journal of Medicinal Plants Research 3:1222-1239.

Kawiak, A., Królica, A. and Lojkowska, E. 2003. Direct regeneration of Drosera from leaf explants and shoot tips. Plant Cell Tissue Organ Cult. 75:175-178.

Kayser, O., Kiderlen, A.F. and Croft, S.L. 2003. Natural products as antiparasitic drugs. Parasitol. Res. 90: S55-S62.

Kim, K.-S. and Jang, G.-W. 2004. Micropropagation of Drosera peltata, a tuberous sundew, by shoot tip culture. Plant Cell Tissue Organ Cult. 77:211-214.

Komaraiah, P., Kavi Kishor, P.B. and Ramkrishna, S.V. 2001. Production of plumbagin from cell cultures of Plumbagorosea L. Biotechnol. Lett. 23:1269-1272.

Komaraiah, P., Ramakrishna, S.V., Reddanna, P. and Kishor, P.B.K. 2003. Enhanced production of plumbagin in immobilized cells of Plumbago rosea by elicitation and in situ adsorption. J. Biotech. 101:181-187.

Krolicka, A., Szpitter, A., Gilgenast, E., Romanik, G., Kaminski, M. and Lojkowska, E. 2008. Stimulation of antibacterial naphthoquinones and flavonoids accumulation in carnivorous plants grown in vitro by addition of elicitors. Enzyme Microb. Tech. 42:216-221.

Kubo, I., Uchida, M. and Klocke, J. 1983. An insect ecdysis inhibitor from the African medicinal plant Plumbago capensis (Plumbaginaceae); a naturally occurring chitin synthetase inhibitor. Agri. Biol. Chem. 47:911-913.

Likhitwitayawuid, K., Kaeamatawong, R., Ruangrungsi, N. and Krungkrai, J. 1998. Antimalarial naphthoquinones from Nepenthes thorelii. Planta Med. 64:237-241.

Lubbe, A. and Verpoorte, R. 2011. Cultivation of medicinal and aromatic plants for specialty industrial materials. Ind. Crop. Prod. 34:785-801.

Luo, Y., Mughal, M.R., Ouyang, T.G., Jiang, H., Luo, W., Yu, Q.S., Greig, N.H. and Mattson, M.P. 2010. Plumbagin promotes the generation of astrocytes from rat spinal cord neural progenitors via activation of the transcription factor Stat3. J. Neurochem. 115:1337-1349.

Mallavadhani, U.V., Sahu, G. and Muralidhar, J. 2002. Screening of Plumbago species for the bio-active marker plumbagin. Pharm. Biol. 40:508-511.

Marczak, L., Kawiak, A., Lojkowska, E. and Stobiecki, M. 2005. Secondary metabolites in in vitro cultured plants of the genus Drosera. Phytochem. Anal. 16:143-149.

Medentsev, A.G. and Akimenko, V.K. 1998. Naphthoquinone metabolites of the fungi. Phytochemistry 47:935-959.

Murashige, T. and Skoog, F. 1962. A revised medium for rapid growth and bio assays with tobacco tissue cultures. Physiol. Plant. 15:473-497.

Nahálka, J., Blanárik, P., Gemeiner, P., Matúsová, E. and Partlová, I. 1996. Production of plumbagin by cell suspension cultures of Drosophyllum lusitanicum Link. J. Biotechnol. 49:153-161.

Nahálka, J., Nahálková, J., Gemeiner, P. and Blanárik, P. 1998. Elicitation of plumbagin by chitin and its release into the medium in Drosophyllum lusitanicum Link suspension cultures. Biotechnol. Lett. 20:841-845.

Ogihara, K., Yamashiro, R., Higa, M. and Yogi, S. 1997. Preparation of napthoquinone derivatives from plumbagin and their ichthyotoxicity. Chemical and Pharmaceutical Bulletin 45:437-445.

Parimala, R. and Sachdanandam, P. 1993. Effect of plumbagin on some glucose metabolising enzymes studied in rats in experimental hepatoma. Mol. Cell. Biochem. 125:59-63.

Putalun, W., Udomsin, O., Yusakul, G., Juengwatanatrakul, T., Sakamoto, S. and Tanaka, 
H. 2010. Enhanced plumbagin production from in vitro cultures of Drosera burmanii using elicitation. Biotechnol. Lett. 32:721-724.

Rates, S.M.K. 2001. Plants as source of drugs. Toxicon 39:603-613.

Ribeiro de Paiva, S., Figueiredo, M.R., Aragão, T.V. and Kaplan, M.A.C. 2003. Antimicrobial activity in vitro of plumbagin isolated from Plumbago species. Memórias do Instituto Oswaldo Cruz 98:959-961.

Rischer, H., Hamm, A. and Bringmann, G. 2002. Nepenthes insignis uses a C2-portion of the carbon skeleton of 1-alanine acquired via its carnivorous organs, to build up the allelochemical plumbagin. Phytochemistry 59:603-609.

Rivadavia, F., Kondo, K., Kato, M. and Hasebe, M. 2003. Phylogeny of the sundews, Drosera (Droseraceae), based on chloroplast and rbcL and nuclear $18 \mathrm{~S}$ ribosomal sequences. Am. J. Bot. 90:123-130.

Rodrigues, S.V., Viana, L.M. and Baumann, W. 2006. UV/Vis spectra and solubility of some naphthoquinones, and the extraction behavior of plumbagin from Plumbago scandens roots in supercritical $\mathrm{CO}_{2}$. Anal. Bioanal. Chem. 385:895-900.

Sankaram, A.V.B, Reddy, V.V.N. and Marthandamurthi, M. $1986 .{ }^{13}$ C NMR Spectra of some naturally ocurring binaphthoquinones and related compounds. Phytochemistry 25:2867-2817.

Sharma, I., Gusain, D. and Dixit, V. 1991. Hypolipidaemic and antiatherosclerotic effects of plumbagin in rabbits. Indian J. Physiol. Pharmacol. 35:10-14.

Shieh, J.M., Chiang, T.A., Chang, W.T., Chao, C.H., Lee, Y.C., Huang, G.Y., Shih, Y.X. and Shih, Y.W. 2010. Plumbagin inhibits TPA-induced MMP-2 and u-PA expressions by reducing binding activities of NF- $\kappa \mathrm{B}$ and AP-1 via ERK signaling pathway in A549 human lung cancer cells. Mol. Cell. Biochem. 335:181-193.

Smelcerovic, A., Spiteller, M. and Zuehlke, S. 2006. Comparison of methods for the exhaustive extraction of hypericins, flavonoids, and hyperforin from Hypericum perforatum L. J. Agr. Food Chem. 54:2750-2753.

Sugie, S., Okamoto, K., Rhaman, K., Tanaka, T., Kawai, K., Yamahara, J. and Mori, H. 1998. Inhibitory effects of plumbagin and juglone on azoxymethane-induced intestinal carcinogenesis in rats. Cancer Lett. 127:177-183.

Szentmihályi, K., Vinkler, P., Lakatos, B., Illes, V. and Then, M. 2002. Rose hip (Rosa canina L.) oil obtained from waste hip seeds by different extraction methods. Bioresource Technol. 82:195-201.

Tandon, V.K., Yadav, D.B., Singh, R.V., Chaturvedic, A.K. and Shuklac, P.K. 2005. Synthesis and biological evaluation of novel (L)- $a$-amino acid methyl ester, heteroalkyl, and aryl substituted 1,4-naphthoquinone derivatives as antifungal and antibacterial agents. Bioorg. Med. Chem. Lett. 15:5324-5328.

Tommasi, F. and Scaramuzzi, F. 2004. In vitro propagation of Ginkgo biloba by using various bud cultures. Biol. Plant. 48:297-300.

Uchendu, E.E., Paliyath, G., Brown, D.C.W. and Saxena, P.K. 2010. In vitro propagation of North American ginseng (Panax quinquefolius L.). In Vitro Cell. Dev. Biol.-Plant DOI: $10.1007 / \mathrm{s} 11627-011-9379-\mathrm{y}$.

Veena, V. and Taylor, C.G. 2007. Agrobacterium rhizogenes: recent developments and promising applications. In Vitro Cell. Dev. Biol.-Plant 43:383-403.

Verma, P.C., Singh, D., Rahman, L., Gupta, M.M. and Banerjee, S. 2002. In vitro-studies in Plumbago zeylanica: rapid micropropagation and establishment of higher plumbagin yielding hairy root cultures. J. Plant Physiol. 159:547-552.

Villavicencio, M.A. and Perez-Escandon, B.E. 1994. Concentracion de plumbagina en Plumbago pulchella Boiss. (Plumbaginaceae) y su efecto en la seleccion de alimento de larvas de Arachnis aulea (Geyer) (Lepidoptera: Arctiidae). Folia Entomologica Mexicana 90:17-24.

Wang, Y.C. and Huang, T.L. 2005. High-performance liquid chromatography for quantification of plumbagin, an anti-Helicobacter pylori compound of Plumbago zeylanica L. J. Chromatogr. A 1094:99-104.

Wink, M., Alfermann, A.W., Franke, R., Wetterauer, B., Distl, M., Windhövel, J., Krohn, 
O., Fuss, E., Garden, H., Mohagheghzadeh, A., Wildi, E. and Ripplinger, P. 2005. Sustainable bioproduction of phytochemicals by plant in vitro cultures: anticancer agents. Plant Gen. Res. 3:90-100.

Wurm, G. and Gurka, H.J. 1986. Untersuchungen an 1,4-Naphthochinonen, 13. Mitt. Neue Synthesen für Plumbagin und Isoplumbagin. Arch. Pharm. 319:190-191.

\section{Tables}

Table 1. The top twelve medicinal and aromatic plants importing and exporting countries, listed according to descending order of average trade volumes between 2004 and 2008 (Adapted from Lubbe and Verpoorte, 2011).

\begin{tabular}{|c|c|c|c|c|c|}
\hline \multicolumn{3}{|c|}{ 2004-2008 Imports } & \multicolumn{3}{|c|}{ 2004-2008 Exports } \\
\hline Country & Tonnes & Value (US\$) ${ }^{\mathrm{a}}$ & Country & Tonnes & Value (US\$) $)^{\mathrm{a}}$ \\
\hline USA & 62038 & 220638 & China & 204082 & 348821 \\
\hline Hong Kong & 48168 & 167298 & India & 47551 & 93450 \\
\hline Germany & 47814 & 139684 & Mexico & 38262 & 38081 \\
\hline Rep. Korea ${ }^{b}$ & 34732 & 47563 & Egypt & 24600 & 34151 \\
\hline Japan & 27937 & 118514 & Germany & 18076 & 99349 \\
\hline China & 27754 & 34808 & Poland $^{\mathrm{b}}$ & 15775 & 47183 \\
\hline France & 20336 & 72471 & Hong Kong & 15011 & 81698 \\
\hline UK & 17895 & 51006 & USA & 13919 & 95704 \\
\hline Spain $^{\text {b }}$ & 15670 & 44337 & Chile $^{b}$ & 11627 & 27656 \\
\hline Italy & 13436 & 59582 & Morocco $^{b}$ & 10796 & 19463 \\
\hline Malaysia & 11348 & 44058 & Bulgaria & 9338 & 21088 \\
\hline Pakistan & 11045 & 4733 & Albania & 7770 & 17084 \\
\hline
\end{tabular}

${ }^{\mathrm{a}}$ In thousands of US dollars.

${ }^{\mathrm{b}}$ Average from 2004 to 2007; data from 2008 not available.

Table 2. Extraction parameters of plumbagin from $D$. intermedia using different extraction methods (Grevenstuk et al., 2012).

\begin{tabular}{lccc}
\hline Method & Extraction yield & \multicolumn{2}{c}{ Plumbagin content } \\
\cline { 3 - 4 }$(\mathrm{mg}$ extract/g FW $)$ & $(\mathrm{mg} / \mathrm{g} \mathrm{FW})$ & $(\mathrm{mg} / \mathrm{g} \mathrm{DW})^{*}$ \\
\hline Maceration & $5.23 \pm 0.61 \mathrm{a}$ & $2.21 \pm 0.17 \mathrm{c}$ & $17.51 \pm 1.40 \mathrm{c}$ \\
SE & $5.63 \pm 0.14 \mathrm{a}$ & $2.67 \pm 0.04 \mathrm{~b}$ & $21.18 \pm 0.44 \mathrm{~b}$ \\
UAE & $5.14 \pm 0.54 \mathrm{a}$ & $3.12 \pm 0.05 \mathrm{a}$ & $24.78 \pm 0.55 \mathrm{a}$ \\
SFE & $5.93 \pm 0.44 \mathrm{a}$ & $2.54 \pm 0.14 \mathrm{bc}$ & $20.15 \pm 1.13 \mathrm{bc}$ \\
\hline
\end{tabular}

Values represent mean \pm standard error of 3 repetitions. For each parameter values with different letters are significantly different at $P<0.05$ according to Duncan's multiple range test. *FW:DW ratio of D. intermedia cultures $=7.9 \pm 0.13: 1$.

Table 3. Purification of plumbagin from $D$. intermedia extracts using SPE (Grevenstuk et al., 2012).

\begin{tabular}{lccc}
\hline Method & SPE yield (mg product/mg extract) & Purity $(\%)$ & SPE recovery (\%) \\
\hline Maceration & $0.37 \pm 0.03 \mathrm{~b}$ & $94.30 \pm 1.80 \mathrm{~b}$ & $82.58 \pm 2.31 \mathrm{a}$ \\
SE & $0.37 \pm 0.01 \mathrm{~b}$ & $99.91 \pm 0.09 \mathrm{a}$ & $78.49 \pm 2.13 \mathrm{a}$ \\
UAE & $0.54 \pm 0.06 \mathrm{a}$ & $99.51 \pm 0.49 \mathrm{a}$ & $86.31 \pm 2.40 \mathrm{a}$ \\
SFE & $0.32 \pm 0.04 \mathrm{~b}$ & $83.90 \pm 3.23 \mathrm{c}$ & $71.58 \pm 3.44 \mathrm{~b}$ \\
\hline
\end{tabular}

Values represent mean \pm standard error of 3 repetitions. For each parameter values with different letters are significantly different at $P<0.05$ according to Duncan's multiple range test. 


\section{Figures}
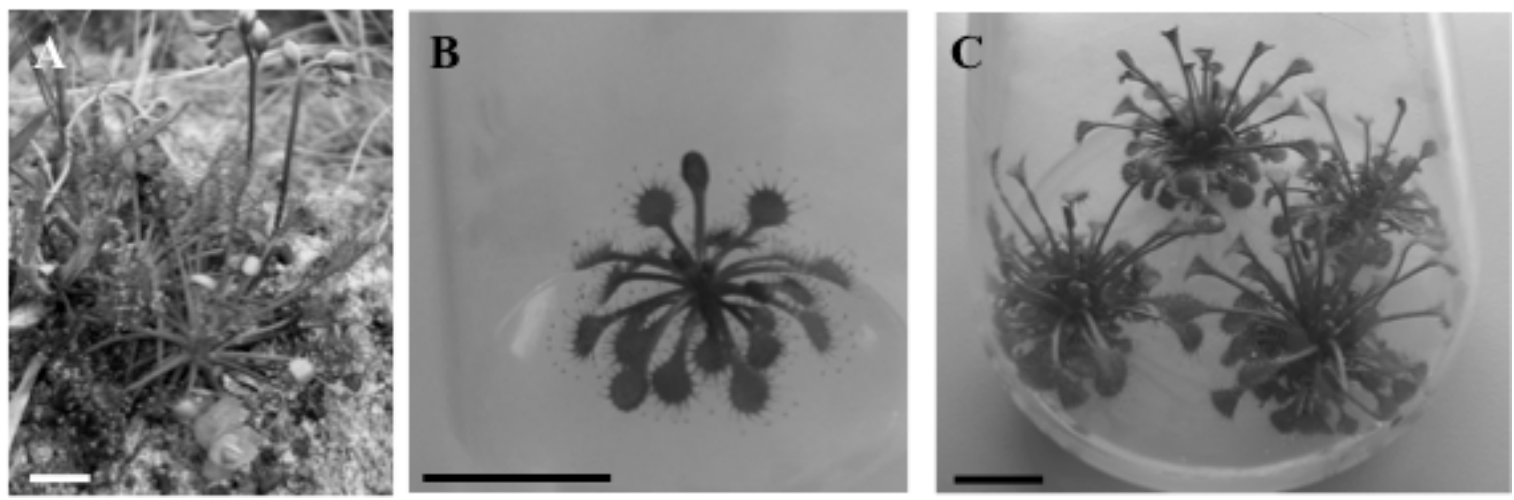

Fig. 1. Micropropagation of D. intermedia: A - field specimen, B - seedling explant, C shoots produced in $1 / 4 \mathrm{MS}$ after 8 weeks of culture.<smiles>CC1=CC(=O)c2c(O)cccc2C1=O</smiles>

Fig. 2. Structure of plumbagin.

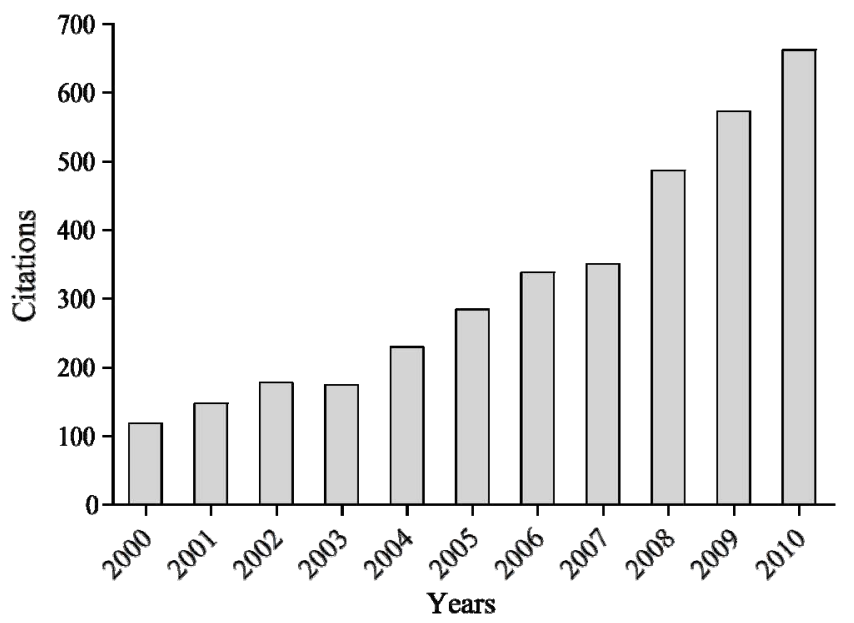

Fig. 3. Citation number in the period of 2000-2010 of published items with the following search criteria: topic="plumbagin", areas="pharmacology and pharmacy". Citation report generated by Web of Science (ISI Web of Knowledge). 


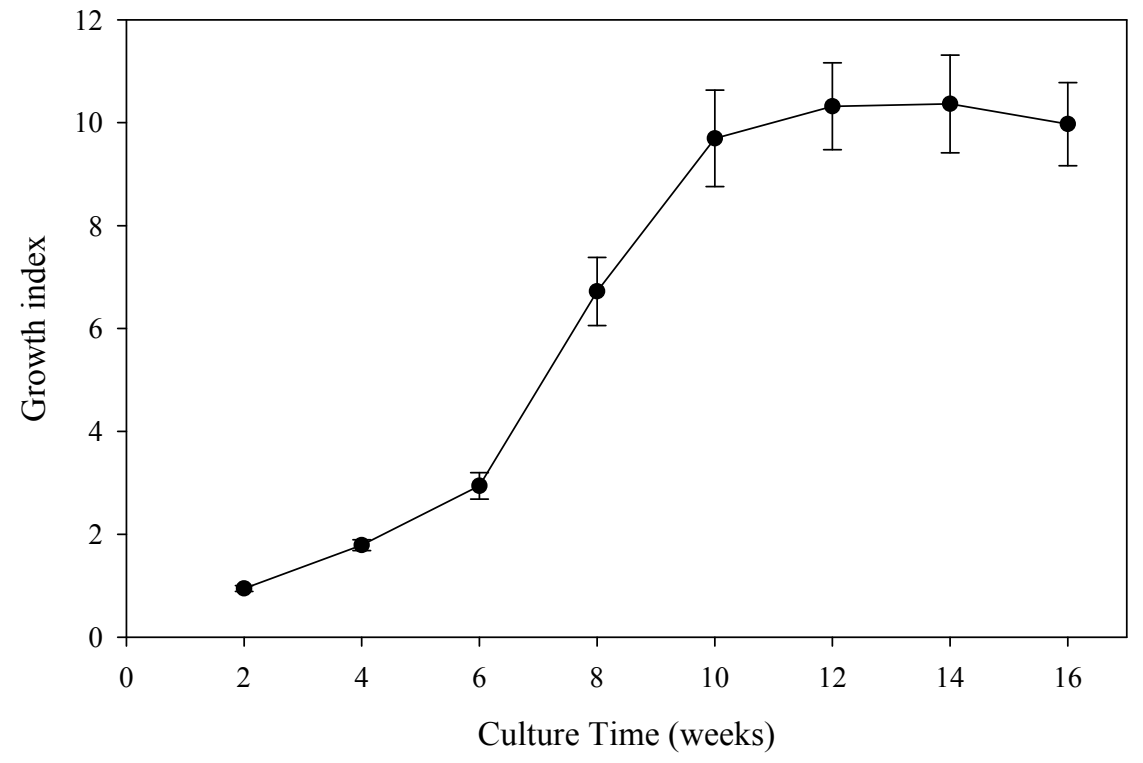

Fig. 4. Growth index of $D$. intermedia cultures during a 16 weeks culture period. The culture growth index was calculated as the fresh weight gain divided by the culture's initial fresh weight and was registered at 2 week intervals. 